\title{
GMR
}

\section{Association between HLA-DRB1 alleles and tuberculosis: a meta-analysis}

\author{
B.F. Chen ${ }^{1}$, R. Wang ${ }^{2}$, Y.J. Chen ${ }^{1}$, Y. Zhu ${ }^{1}$, L. Ding ${ }^{1}$ and Y.F. Wen ${ }^{1}$ \\ ${ }^{1}$ School of Public Health, Wannan Medical College, Wuhu, China \\ ${ }^{2}$ Department of Health Inspection and Supervision, Wuhu Health Bureau, Wuhu, \\ China \\ Corresponding authors: Y.F. Wen / B.F. Chen \\ E-mail: wyf@wnmc.edu.cn / chenbf1980@163.com
}

Genet. Mol. Res. 14 (4): 15859-15868 (2015)

Received June 13, 2015

Accepted September 11, 2015

Published December 1, 2015

DOI http://dx.doi.org/10.4238/2015.December.1.37

ABSTRACT. Although a number of studies have reported that human leukocyte antigen (HLA)-DRB1 alleles may be correlated with tuberculosis (TB), most were based on small samples or inconsistent and unclear results. Here, we present a meta-analysis to investigate the relationship between HLA-DRB1 alleles and TB susceptibility. We gathered relevant information from published studies on the association between HLA-DRB1 alleles and TB susceptibility through a systematic research. Data from eligible fifteen studies were included in the meta-analyses. Each dataset was statistically analyzed to evaluate the HLA-DRB1 alleles by calculating the respective odds ratios (ORs) and 95\% confidence intervals (Cls). The results revealed that frequencies of two DRB1 alleles were significantly decreased in TB: DRB1*03 $(\mathrm{P}=0.016, \mathrm{OR}=0.78,95 \% \mathrm{Cl}=0.67-0.95)$ and $\mathrm{DRB} 1{ }^{*} 07(\mathrm{P}=0.017, \mathrm{OR}=0.81,95 \% \mathrm{Cl}=0.68-0.96)$. Thus, our data indicate that $\mathrm{DRB} 1{ }^{*} 03$ and $\mathrm{DRB} 1{ }^{*} 07$ may provide protective effects against TB susceptibility. However, well-designed studies with large sample sizes are required for better understanding of this association.

Key words: Human leukocyte antigen-DRB1; Tuberculosis; Meta-analysis 


\section{INTRODUCTION}

Tuberculosis (TB), primarily caused by Mycobacterium tuberculosis (M. tuberculosis), is a significant health burden worldwide. Approximately one-third of the global population is estimated to be infected by M. tuberculosis with 9.4 million new cases and 1.7 million fatalities estimated each year (Zumla et al., 2013). Pathogenesis of TB is a complex process that is not completely understood. M. tuberculosis infection in humans may be eradicated by host response or remain latent; only $10 \%$ of infected individuals may develop the disease during their life span (Azad et al., 2012).

Human leukocyte antigen (HLA) system is the major histocompatibility complex in humans and is known to play an important role in controlling immune response (Benacerraf, 1981; Bjorkman et al., 1987). The HLA region is located in the short arm of chromosome 6 and it is separated into three sub-regions: the HLA class I region- encoding for HLA-A, -B, and -C antigens; the HLA class II region encoding for HLA-DR, -DQ, and -DP antigens; and the HLA class III region encoding for the second and fourth component of complements $\mathrm{C} 2$ and $\mathrm{C} 4$, factor $\mathrm{B}$, tumor necrosis factors $\alpha$ and $\beta$, heat shock protein 70, and 21-hydroxylase. The class I and class Il genes code for cell-surface glycoproteins, which play a fundamental role in the homeostasis of the immune response by presenting antigenic peptides to cluster of differentiation 81 (CD81) and CD41 T cells (Accolla et al., 1995). The HLA-DRB1 genes have the highest number of polymorphism in the HLA class II; these allelic variants are known to vary in their preferential binding of antigenic peptides and are associated with resistance and susceptibility to numerous infectious diseases including TB (Hill, 1998).

While a number of studies have focused on the association of certain DRB1 alleles with TB susceptibility, the results have been conflicting. Some studies have identified a positive association between the DRB1 alleles and TB across geographical locations- DRB1*15 in the Sahariya tribe (Mishra et al., 2014), DRB1*0801 and DRB1*0803 in Kazakhstan (Kuranov et al., 2014), DRB1*15 in Chinese (Shi et al., 2011), DRB1*14 in Portuguese (Duarte et al., 2011), DRB1*0803 in Korean (Kim et al., 2005), DRB1*16 in Polish (Dubaniewicz et al., 2000), DRB1*04 and DRB1*11 in Syrian (Harfouch and Daher, 2008), and DRB1*07 in Iranian populations (Amirzargar et al., 2004). However, other studies have reported no association. Yuliwulandari found that DRB1*1202 showed a protective effect against TB in Indonesia (Yuliwulandari et al., 2010), whereas Vejbaesya (Vejbaesya et al., 2002) reported no significant difference in DRB1 alleles between TB patients and controls in Thailand. Another report from Brazil showcased overrepresention of DRB $1^{*} 04$ and under-representation of DRB1*15 in TB patients (Lucena et al., 2010).

Although most of the previous studies have disagreed over the contribution of the DRB1 alleles to TB, the small sample sizes and inadequate statistical power in these studies were controversial rather than conclusive. Therefore, in an effort to clarify the association of the DRB1 alleles of the HLA-II genes and TB risk, the current meta-analysis was performed to summarize the available data.

\section{MATERIAL AND METHODS}

\section{Literature and search strategy}

This study was designed according to the Meta-analysis of Observational Studies in Epidemiology (MOOSE) guidelines (Stroup et al., 2000). PubMed and Embase were searched 
for studies associated with the following medical subject heading (MeSH) terms: i) 'Tuberculosis' or 'TB' AND ii) 'human leukocyte antigen,' 'HLA' or 'DRB1'. The search was limited to reports in English. All the publications were searched until October 30, 2014. In addition, the reference lists of the retrieved studies and reviews were manually searched for additional studies that were not identified in the initial search.

\section{Inclusion and exclusion criteria}

Studies were selected according to the following inclusion criteria: i) An independent case-control design; ii) primary study (not re-analysis or review) in English; iii) sufficient information to calculate the odds ratio (OR); iv) unrelated cases and controls. Studies were excluded based on the following features: i) Incomplete raw data; ii) repetitive reporting of a dataset (for which the most recent study was selected for inclusion); iii) studies without detailed HLA-DRB1 alleles.

\section{Data extraction}

Two investigators (Chen and Wang) independently reviewed and extracted the information from all the eligible studies according to the inclusion and exclusion criteria listed above. Disagreements were resolved by discussion and by consulting a third investigator (Wen). The following information was collected from each of the selected studies: First author's surname, publication year, country of study population, detection assay type and numbers of cases and controls for each genetic variation.

\section{Statistical analysis}

The effect of HLA-DRB1 alleles on TB was measured by using the Mantel-Haenszel method to calculate the combined ORs and $95 \%$ confidence intervals (Cls). Initially, numerical data was extracted directly and converted into a numerical statistical value by multiplying the frequency data and the total sample number. Subsequently, Review Manager software (version 5.2; http://www.cochrane.org) was used for the meta-analysis. The heterogeneity among different studies was estimated by chi-square $\left(X^{2}\right)$ test, Q-statistic test and $I^{2}$ test (Higgins et al., 2002). When the $\mathrm{P}$ value for heterogeneity (Ph) was greater than 0.10 or $\mathrm{I}^{2}<$ $50 \%$, the heterogeneity was considered statistically insignificant and the fixed-effects model was applied (Mantel and Haenszel, 1959); otherwise, the random-effects model was used (DerSimonian and Laird, 1986). When the $\mathrm{P}$ value for comparability $(\mathrm{Pc})$ was greater than 0.05 (two-tailed test) and the $95 \% \mathrm{Cl}$ of OR was exclusive of 1.0 , the value was considered statistically significant. In order to avoid type II errors, Bonferroni adjustment of $P$ values was not employed for multiple comparisons. An estimate of potential publication bias were carried out by funnel plot when at least five papers were included. The funnel plot asymmetry were also assessed by Begg's rank correlation test and Egger's linear regression test, $\mathrm{P}<0.05$ was considered representative of statistically significant publication bias. (Egger et al., 1997; Nakagawa, 2004;). The Begg's and Egger's tests were carried out by R software, version 3.2.2 (http://r-project.org/). 


\section{RESULTS}

\section{Characteristics of included studies}

A total of 229 potentially relevant studies were identified by the initial database search; 56 studies were overlapping and 150 were excluded by subsequent screening of title and abstract. Eventually, 23 studies that investigate the association of the HLA-DRB1 alleles with TB patients were shortlisted. Following restricted selection according to the inclusion and exclusion criteria, 15 studies were finally selected for inclusion in the meta-analysis (Figure 1). The characteristics of the included studies are presented in Table 1.

\section{Meta-analyses of HLA-DRB1 alleles and their correlation to TB}

Data on 1,640 cases and 2,024 controls from 15 case-control studies were included for analysis of the association between HLA-DRB1 alleles and TB. A total of 13 different DRB1 alleles, namely HLA-DRB1*01, 03, 04, 07, 08, 09, 10, 11, 12, 13, 14, 15, and 16 were assayed. While one of the included study did not report the alleles DRB1*01 and DRB1*13 (Ravikumar et al., 1999), another did not report the DRB1*04 allele (Shi et al., 2011). Pooled ORs and $95 \%$ Cls indicated that two HLA variants namely DRB1*03 and DRB1*07 showed a protective effect against TB (P $\leq 0.05)$. A fixed-effects model was used based on the detected heterogeneity in the test results $\left(\mathrm{Ph}=0.768, \mathrm{I}^{2}=0 \%\right.$; and $\mathrm{Ph}=0.416, \mathrm{I}^{2}=3.1 \%$, respectively). Other HLA-DRB1 alleles showed no statistically significant association with TB risk in the present meta-analysis. The results are presented in Table 2.

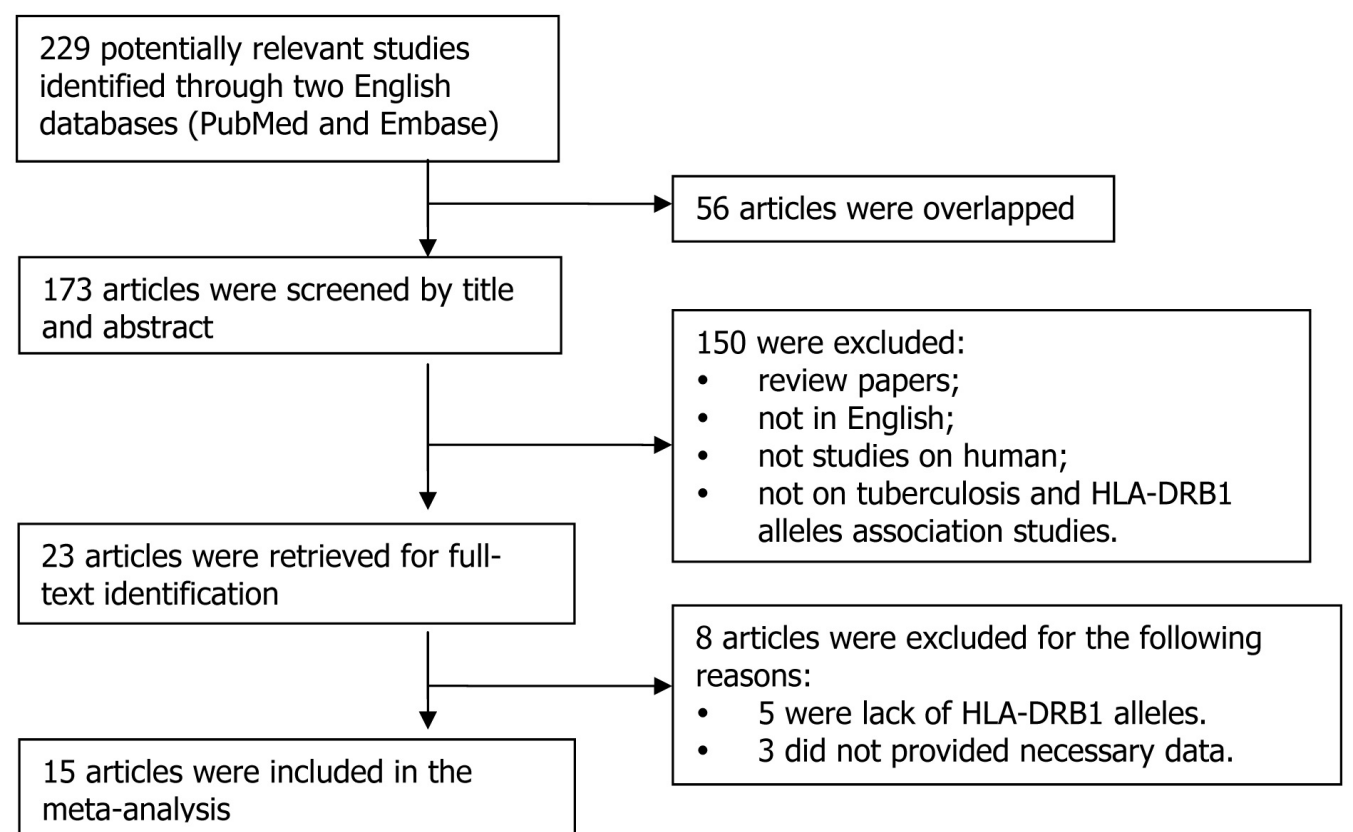

Figure 1. Flow chart of study selection based on the inclusion and exclusion criteria. HLA, human leukocyte antigen. 


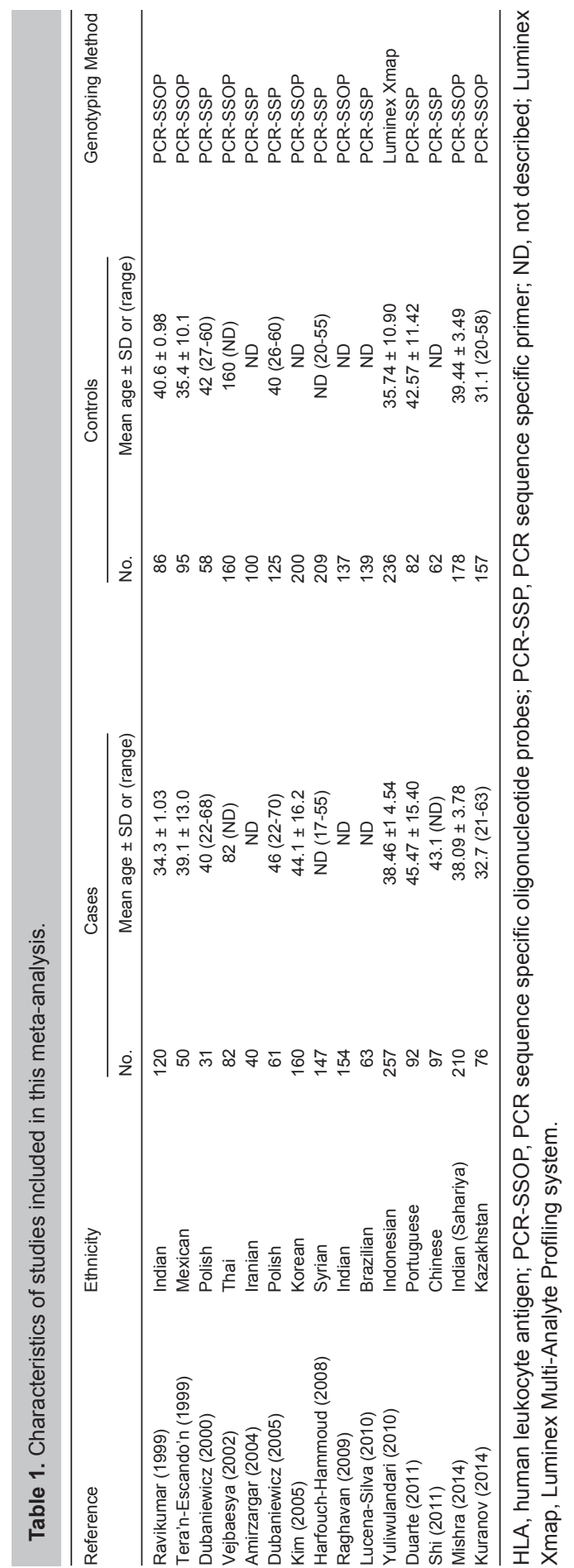




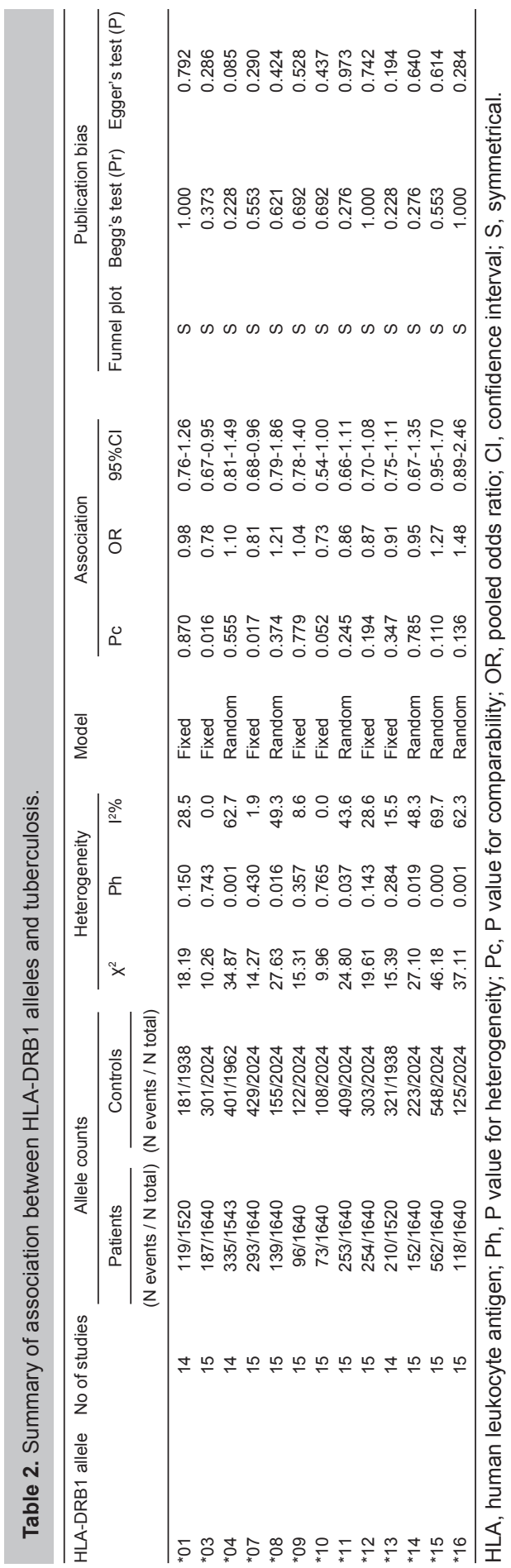




\section{Sensitivity analysis and publication bias}

In order to test the influence of individual datasets, the pooled OR was calculated excluding one study at a time. It was found that in majority of the cases, the corresponding pooled ORs were not materially altered (data not shown). All the funnel plots were inspected for geometrical symmetry and were found to be roughly symmetrical. The funnel plots of DRB $1{ }^{*} 01$ and DRB $1{ }^{*} 07$ are presented in Figure 2. These plots confirmed the absence of publication bias, which was consistent with the outcomes of the Begg's and Egger's tests $(P>0.05)$.
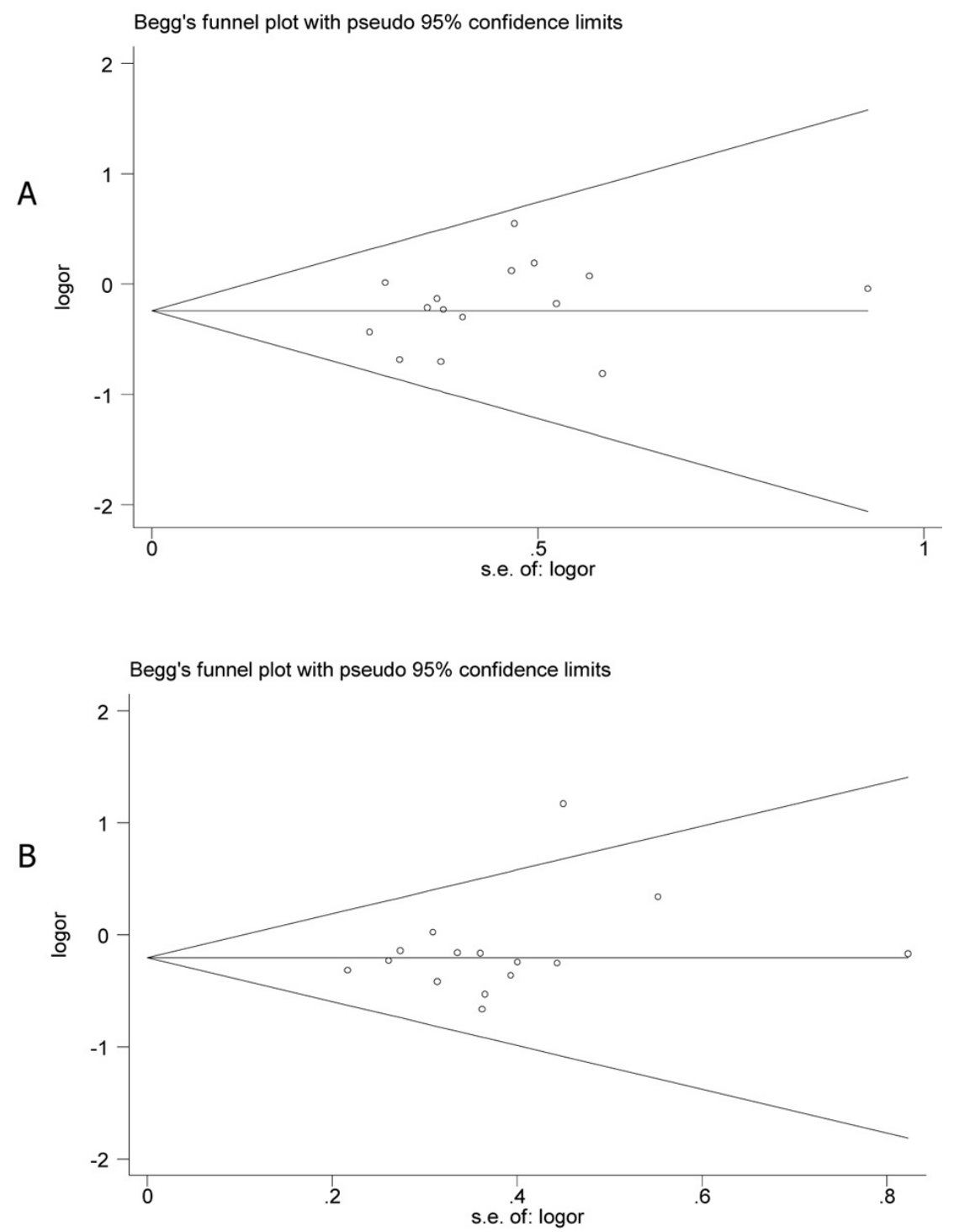

Figure 2. Begg's funnel plots for publication bias in selection of studies on the HLA-DRB1 alleles. (A) HLA-DRB1 ${ }^{*} 03$, (B) HLA-DRB1*07. HLA, human leukocyte antigen; OR, odds ratio; SE, standard error. 


\section{DISCUSSION}

The outcome of TB is widely known to be regulated by the environment as well as bacterial and host genetic components. A number of studies have confirmed that HLA genes play a critical role in the development of TB. HLA genes are considered important candidate biomarkers for susceptibility and/or resistance to infectious diseases because they exhibit a markedly high degree of polymorphisms and govern the host immune response of against invading pathogens. Regardless of extensive research to determine the function of HLA-DRB1 alleles in the pathogenesis of TB, the findings thus far have been inconsistent and controversial. Therefore, we have performed a comprehensive meta-analysis to investigate this association, the first of its type to the best of our knowledge. Although earlier studies have shown that $\mathrm{DRB} 1^{*} 03$ and $\mathrm{DRB}{ }^{*} 07$ were not associated with TB susceptibility, this meta-analysis revealed that these HLA-DRB1 alleles may have a negative association with TB, indicative of a possible protective role against TB.

In different populations, specific HLA-DRB1 alleles have been associated with TB. For example, DRB1*15 has been reported to be associated with the susceptibility of TB in various ethnic groups such as Indian (Rajalingam et al., 1996; Ravikumar et al., 1999; Stroup DF et al., 2000; Sriram et al., 2001; Mishra et al., 2014; Kuranov et al., 2014), Mexican (Teran et al., 1999), Chinese (Shi et al., 2011), Polish (Dubaniewicz et al., 2005) and Korean (Kim et al., 2005). DRB1*16 is reported to have a positive association with the susceptibility to tuberculosis in Polish population (Dubaniewicz et al., 2005), but a negative association in the Sahariya tribe of North Central India (Mishra et al., 2014). DRB1*12 has been reported in the Indonesian population (Yuliwulandari et al., 2010), DRB1*13 in the Polish (Dubaniewicz et al., 2000), and South African populations (Lombard Z et al., 2006), and DRB1*14 in the Iranian (Mahmoudzadeh et al., 2003) and Portuguese populations (Duarte et al., 2011). The differences in the HLA-DRB alleles between the populations can be partly explained by the variation in the technique used, limited sample size and linkage disequilibrium.

These results should be interpreted with caution due to certain limitations. The possibility of selection bias cannot be strictly excluded as only published studies written in English and cited by the PubMed and Embase databases were included for analysis. However, no evidence of publication bias was observed. In addition, different ethnic populations and sources of controls may contribute to bias in data. Further, the environment factors contributing to susceptibility of TB were not accounted since the information from the included studies was limited. Finally, meta-analysis remains a retrospective investigation that is subject to the methodological deficiencies of the included studies. In order to overcome these limitations, future case-control studies should focus on the clinical or laboratory characteristics in a large cohort of TB patients from different ethnicities.

\section{Conflicts of interest}

The authors declare no conflict of interest.

\section{ACKNOWLEDGMENTS}

Research supported by the University Natural Science Foundation of Anhui Province. Grant \#KJ2014A272. 


\section{REFERENCES}

Accolla RS, Adorini L, Sartoris S, Sinigaglia F, et al. (1995). MHC: orchestrating the immune response. Immunol. Today 16: 8-11.

Amirzargar AA, Yalda A, Hajabolbaghi M, Khosravi F, et al. (2004). The association of HLA-DRB, DQA1, DQB1 alleles and haplotype frequency in Iranian patients with pulmonary tuberculosis. Int. J. Tuberc. Lung Dis. 8: 1017-1021.

Azad AK, Sadee W and Schlesinger LS (2012). Innate immune gene polymorphisms in tuberculosis. Infect Immun. 80: 3343-3359.

Benacerraf B (1981). Role of MHC gene products in immune regulation. Science 212: 1229-1238.

Bjorkman PJ, Saper MA, Samraoui B, Bennett WS, et al. (1987). The foreign antigen binding site and T cell recognition regions of class I histocompatibility antigens. Nature 329: 512-518.

DerSimonian R and Laird N (1986). Meta-analysis in clinical trials. Controlled Clin. Trials 7: 177-188.

Duarte R, Carvalho C, Pereira C, Bettencourt A, et al. (2011). HLA class II alleles as markers of tuberculosis susceptibility and resistance. Rev. Port. Pneumol. 17: 15-19.

Dubaniewicz A, Lewko B, Moszkowska G, Zamorska B, et al. (2000). Molecular subtypes of the HLA-DR antigens in pulmonary tuberculosis. Int. J. Infect. Dis. 4: 129-133.

Dubaniewicz A, Moszkowska G and Szczerkowska Z (2005). Frequency of DRB1-DQB1 two-locus haplotypes in tuberculosis: preliminary report. Tuberculosis 85: 259-267.

Egger M, Davey Smith G, Schneider M and Minder C (1997). Bias in meta-analysis detected by a simple graphical test. BMJ 315: 629-634.

Harfouch-Hammoud El and Daher NA (2008). Susceptibility to and severity of tuberculosis is genetically controlled by human leukocyte antigens. Saudi Med. J. 29: 1625-1629.

Higgins JP and Thompson SG (2002). Quantifying heterogeneity in a meta-analysis. Stat. Med. 21: 1539-1558.

Hill AV (1998). The immunogenetics of human infectious diseases. Annu. Rev. Immunol. 16: 593-617.

Kim HS, Park MH, Song EY, Park H, et al. (2005). Association of HLA-DR and HLA-DQ genes with susceptibility to pulmonary tuberculosis in Koreans: preliminary evidence of associations with drug resistance, disease severity, and disease recurrence. Hum. Immunol. 66: 1074-1081.

Kuranov AB, Kozhamkulov UA, Vavilov MN, Belova ES, et al. (2014). HLA-class II alleles in patients with drug-resistant pulmonary tuberculosis in Kazakhstan. Tissue Antig. 83: 106-112.

Lombard Z, Dalton DL, Venter PA, Williams RC, et al. (2006). Association of HLA-DR, -DQ and vitamin D receptor alleles and haplotypes with tuberculosis in the Venda of South Africa. Hum. Immunol. 67: 643-654.

Lucena-Silva N, Baliza MD, Martins AE, Deghaide NH, et al. (2010). Relatedness and HLA-DRB1 typing may discriminate the magnitude of the genetic susceptibility to tuberculosis using a household contact model. J. Epidemiol. Community Health 64: 513-517.

Mahmoudzadeh-Niknam H, Khalili G and Fadavi P (2003). Allelic distribution of human leukocyte antigen in Iranian patients with pulmonary tuberculosis. Hum. Immunol. 64: 124-129.

Mantel $\mathrm{N}$ and Haenszel W (1959). Statistical aspects of the analysis of data from retrospective studies of disease. J. Natl. Cancer Inst. 22: 719-748.

Mishra G, Kumar N, Kaur G, Jain S, et al. (2014). Distribution of HLA-A B and DRB1 alleles in Sahariya tribe of North Central India: an association with pulmonary tuberculosis. Infect. Genet. Evol. 22: 175-182.

Nakagawa S (2004). A farewell to Bonferroni: the problems of low statistical power and publication bias. Behav. Ecol. 15: 1044-1045.

Raghavan S, Selvaraj P, Swaminathan S, Alagarasu K, et al. (2009). Haplotype analysis of HLA-A, -B antigens and -DRB1 alleles in south Indian HIV-1-infected patients with and without pulmonary tuberculosis. Int. J. Immunogenet. 36: 129-33.

Rajalingam R, Mehra NK, Jain RC, Myneedu VP, et al. (1996). Polymerase chain reaction-based sequence specific oligonucleotide hybridization analysis of HLA class II antigens in pulmonary tuberculosis: relevance to chemotherapy and disease severity. J. Infect. Dis. 173: 669-676.

Ravikumar M, Dheenadhayalan V, Rajaram K, Lakshmi SS, et al. (1999). Associations of HLA-DRB1, DQB1 and DPB1 alleles with pulmonary tuberculosis in south India. Tuber. Lung Dis. 79: 309-317.

Shi GL, Hu XL, Yang L, Rong CL, et al. (2011). Association of HLA-DRB alleles and pulmonary tuberculosis in North Chinese patients. Genet. Mol. Res. 10: 1331-1336.

Sriram U, Selvaraj P, Kurian SM, Reetha AM, et al. (2001). HLA-DR2 subtypes \& immune responses in pulmonary tuberculosis. Indian J. Med. Res. 113: 117-124.

Stroup DF, Berlin JA, Morton SC, Olkin I, et al. (2000). Meta-analysis of observational studies in epidemiology: a proposal for reporting. Meta-analysis of Observational Studies in Epidemiology (MOOSE) group. JAMA 283: 2008-2012. 
Teran-Escandon D, Teran-Ortiz L, Camarena-Olvera A, Gonzalez-Avila G, et al. (1999). Human leukocyte antigen-associated susceptibility to pulmonary tuberculosis: molecular analysis of class II alleles by DNA amplification and oligonucleotide hybridization in Mexican patients. Chest 115: 428-433.

Vejbaesya S, Chierakul N, Luangtrakool K, Srinak D, et al. (2002). Associations of HLA class II alleles with pulmonary tuberculosis in Thais. Eur. J. Immunogenet. 29: 431-434.

Yuliwulandari R, Sachrowardi Q, Nakajima H, Kashiwase K, et al. (2010). Association of HLA-A, -B and -DRB1 with pulmonary tuberculosis in western Javanese Indonesia. Hum. Immunol. 71: 697-701.

Zumla A, George A, Sharma V, Herbert N, et al. (2013). WHO's 2013 global report on tuberculosis: successes, threats, and opportunities. Lancet 382: 1765-1767. 